\title{
Photopigment Transmittance Imaging of the Primate Photoreceptor Mosaic
}

\author{
Orin S. Packer, David R. Williams, and David G. Bensinger \\ Center for Visual Science, University of Rochester, Rochester, New York 14627
}

We introduce a new technique for classifying many photoreceptors simultaneously in fresh, excised primate retina on the basis of their absorptance spectra. Primate retina is removed from the pigment epithelium and illuminated under a microscope from the same direction as in the intact eye. To facilitate the guiding of light into the receptor outer segments, the optical axes of the photoreceptors are oriented parallel to the optical axis of the microscope. Photoreceptor outer-segment tips are imaged on a charge-coupled device array, which provides radiometric measurements of the light passing through each photoreceptor. These images are acquired sequentially at three wavelengths chosen to maximize the absorptance differences among the three cone photopigments. After the photopigment is bleached, a second set of three images is acquired. The ratios of the images before and after bleaching at each wave- length are photopigment transmittance maps of the retina. These are combined into a single trichromatic image showing the distribution of photopigment if the retina could be viewed directly in white light without bleaching. We have found patches of receptors in peripheral macaque retina where the measured absorptance at the wavelength of maximum absorptance is consistent with the predicted axial absorptance of the photopigment. The cones in these patches cluster into two groups corresponding to the middle wavelength-sensitive ( $n=53$, mean absorptance $=0.28$ ) and the long wavelength-sensitive ( $n=63$, mean absorptance $=0.30$ ) cones. The mean absorptances of 273 macaque and 183 human rods were 0.51 and 0.41 , respectively.

Key words: photoreceptors; cones; rods; photopigment; topography; primate
Although color vision is mediated by three photoreceptor classes, we have an incomplete idea about how the short (S), middle (M), and long $(\mathrm{L})$ wavelength-sensitive cones are arranged across the retina. $S$ cones are absent in the foveal center and form a sparse, nonrandom packing arrangement in peripheral retina (Marc and Sperling, 1977; Williams et al., 1981; de Monasterio et al., 1985; Ahnelt et al., 1987; Wikler and Rakic, 1990; Curcio et al., 1991). L-to-M cone ratios of 1 in the monkey and 2 in the human and a random arrangement of $L$ and $M$ cones are consistent with available data (Brown and Wald, 1963, 1964; MacNichol, 1964; Marks et al., 1964; Walraven, 1974; Smith and Pokorny, 1975; Bowmaker and Dartnall, 1980; Bowmaker et al., 1980a,b, 1983, 1991; Dartnall et al., 1983a,b; MacNichol et al., 1983; Baylor et al., 1987; Harosi, 1987; Schnapf et al., 1987, 1988; Cicerone and Nerger, 1989; Vimal et al., 1989; Wesner et al., 1991; Mollon and Bowmaker, 1992; Cicerone and Otake, 1993; Gowdy et al., 1993; Cicerone et al., 1994; Hagstrom et al., 1994, 1995). Because of sampling limitations, however, even these general conclusions must be accepted with some caution.

The ideal technique for studying photoreceptor topography should label simultaneously every photoreceptor in a patch of undisturbed living retina on the basis of measurements linked directly to photopigment spectral properties. Axial microspectrophotometry (MSP) meets these requirements most closely.

Denton and Wyllie (1955) illuminated excised frog retina and photographically measured the proportion of light transmitted

\footnotetext{
Received Nov. 13, 1995; revised Dec. 26, 1995; accepted Jan. 4, 1996.

This research was supported by Grants EY09625 and EY01319 from National Institutes of Health. We thank Bill Haake, Al Russell, and Andrew Asproulis for technical assistance, and Walt Makous for helpful discussions.

Correspondence should be addressed to Orin S. Packer, Center for Visual Science, 274 Meliora Hall, University of Rochester, Rochester, NY 14627.

Copyright $\odot 1996$ Society for Neuroscience $\quad 0270-6474 / 96 / 162251-10 \$ 05.00 / 0$
}

axially through the photopigment (transmittance) as a function of wavelength. They distinguished photoreceptor types directly on the spectral properties of the photopigments, preserving retinal topography. Unfortunately, when applied to primate photoreceptors (Brown and Wald, 1963, 1964; MacNichol, 1964; Marks et al., 1964), axial MSP yielded low (typically <0.05) photopigment absorptances (1-transmittance) and unreliable spectral sensitivities. Even the most recent axial study (Mollon and Bowmaker, 1992) reported low absorptances.

Color-matching (Burns and Elsner, 1985; Burns et al., 1987), retinal densitometry (Ripps and Weale, 1963; Rushton and Henry, 1968; Alpern et al., 1971; Smith et al., 1983; van Norren and van de Kraats, 1989; Elsner et al., 1993), and photopigmentspecific density (Bowmaker et al., 1980a; Harosi, 1987) combined with outer-segment length (Yuodelis and Hendrickson, 1986) suggest that approximately half of the incident photons are absorbed by photopigment. Thus, values reported by axial MSP are as much as 10 times too low. Possible reasons for this discrepancy include changes in optical properties or reduction in outersegment length during tissuc preparation (Liebman, 1972).

Our goal is to apply recent advances for maintaining primate retina in vitro (Ames and Nesbett, 1981; Dacey, 1989) and a new generation of high resolution, highly linear imaging detectors to the classic technique of axial MSP. The resulting new technique, photopigment transmittance imaging, should better preserve the optical and physical characteristics of the tissue and maximize the reliability of the measurements of the light being transmitted through the photopigment.

\section{MATERIALS AND METHODS}

Tissue preparation. Macaque monkey (Macaca mulatta and Macaca fascicularis) retinas were obtained after acute experiments performed by other investigators after which an overdose of barbiturate anesthesia was 


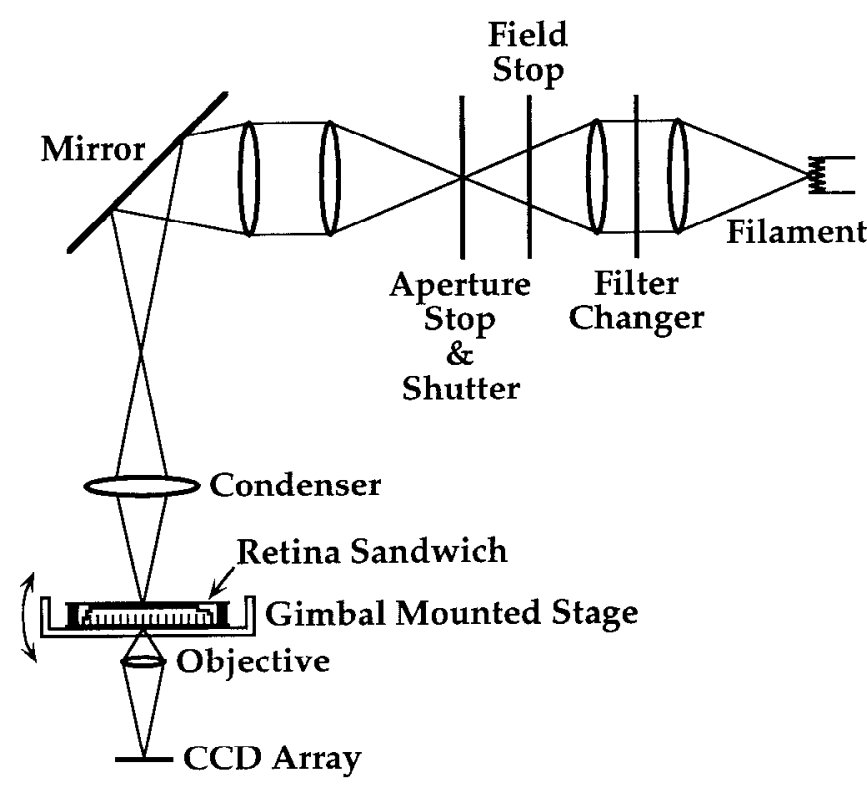

Figure 1. Schematic diagram of the apparatus uscd to image the retina. Light from a filament illuminates a piece of fresh, excised primate retina. Light passing through the retina is then imaged on a CCD. See Materials and Methods (Apparatus) for a complete description.

administered. A single human retina was obtained from the Rochester Eye and Human Parts Bank several hours after the donor died. To minimize the rapid loss in transparency that occurs after normal blood flow ceases (Enoch and Glisman, 1966; Liebman, 1972; Ohzu and Enoch, 1972; Ohzu et al., 1972), the retina was placed in tissue culture immediately after being harvested. To prevent bleaching of the light-sensitive photopigment, all tissue handling was done under infrared (IR) illumination with the aid of IR viewers (FJW Optical, Palatine, IL) mounted on the microscope and near the dissection area. Immediately after enucleation, the front of the eye was removed with sharp scissors. After as much vitreous humor as possible was removed, the retina was placed in a small Petri dish and superfused with artificial cerebral spinal fluid (Ames medium; Sigma, St. Louis, MO) specifically formulated for maintaining retinas in vitro (Ames and Nesbett, 1981). The room temperature (typically $28^{\circ} \mathrm{C}$ ) medium, $\mathrm{pH}$-adjusted to 7.4 , was bubbled continuously with $95 \% \mathrm{O}_{2} / 5 \% \mathrm{CO}_{2}$ as it entered the chamber from one side, flowed across the retina at a rate of $2-3 \mathrm{ml} / \mathrm{min}$, and drained from the opposite side. Keeping the Ames medium at $37^{\circ} \mathrm{C}$ did not produce noticeably better results. A similar preparation maintains the light response of primate photoreceptors for several hours (Dacey, 1989; Dacey and Lee, 1994a,b). An advantage of this technique is that retina not being viewed can be preserved for several hours under conditions as similar as possible to those in vivo.

Each disk of retina used in an experiment was cut from the superfused retina with a sharp trephine (4 $\mathrm{mm}$ diameter) and moved to a dissection dish filled with Ames medium. The retina was teased from the pigment epithelium with a fine forceps and allowed to float frecly. The retina was picked up on a cover glass and mounted between two pieces of cover glass separated by a $100-\mu \mathrm{m}$-thick spacer. This retina sandwich was then placed in a holder attached to the microscope stage. The total time from cessation of superfusion until imaging was -5 min.

Apparatus. Images were collected with an inverted microscope (IMT-2) (Olympus, Lake Success, NY) equipped with modified illumination optics and a charge-coupled device (CCD) camera (Fig. 1). A stepping motor allowed the fine focus of the microscope to be computer-controlled in 1 $\mu \mathrm{m}$ steps. Light from a tungsten-halogen filament was focused by a condenser onto the retina. Exposure duration and wavelength were controlled with a shutter and interference filters $(10 \mathrm{~nm}$ full width at half-maximum) (Corion, Holliston, MA). An aperture stop limited the angular extent of the light cone reaching the retina to $\sim 5^{\circ}$, similar to the angular extent produced by a $2 \mathrm{~mm}$ human pupil. A field stop controlled the diameter of the spot of light imaged on the retina.

The stage to which the retina sandwich was attached incorporated a custom gimbal mount that allowed it to tilt smoothly around two perpendicular axes (see Fig. 1 for one axis), the intersection of which coincided with the center of the field of view. This allowed the retina to remain in focus and stationary when the stage was tilted.

Light was transmitted through the retina in the normal direction and emerged from the photoreceptors where it was collected by the microscope objective (ULWD CD Plan, 20 or $40 \times$, Olympus). These objectives had extra-long working distances and could be adjusted for viewing through glass up to $2 \mathrm{~mm}$ thick. The objective formed an image of the outer segments in the image plane of a CCD camera with the aid of a $2.5 \times$ photographic ocular (not shown) for a total magnification of 50 or $100 \times$. The field of view was $0.66(140 \mu \mathrm{m})$ or $0.33^{\circ}(70 \mu \mathrm{m})$ in diameter. To allow corrections for axial chromatic aberration, the sharpest focus for each objective at each wavelength was calibrated using a fine Ronchi ruling. These calibrations were used hy the computer to refocus the microscope for the sharpest possible image for each combination of wavelength and objective.

Images were collected with a cooled CCD camera (SU-200, Photometrics, Tucson, AZ) fitted with a Kodak KAF1400 CCD chip. The large number of imaging elements $(1320 \times 1035$ pixels, $9 \times 7 \mathrm{~mm}$ active area $)$ made it possible to image large numbers of photoreceptors at high resolution. The high quantum efficiency (33\%) minimized pigment bleaching. The large and linear dynamic intensity range ( 12 bits) allowed reliable discrimination of very low levels of contrast

Optimum wavelengths for maximum cone contrast. Because only enough spectral information was needed to unambiguously distinguish $\mathrm{S}, \mathrm{M}$, and $\mathrm{L}$ cones, we collected images at only three wavelengths. These were chosen to give the highest contrast among the cone photopigment types and were calculated from the cone spectral sensitivities (Baylor et al., 1987) and estimates of the length of the cone outer segments. The optimum wavelength for distinguishing $S$ from $M$ and $L$ cones is $428 \mathrm{~nm}$, whereas the optimum wavelengths for distinguishing $L$ and $M$ cones are 508 and $588 \mathrm{~nm}$. For convenience, we used narrow bandwidth interference filters with maximum transmissions at 440,510 , and $590 \mathrm{~nm}$. The absorption spectra of the interference filters were confirmed with a spectrophotometer.

Photoreceptor alignment. Individual photoreceptors capture the highest proportion of light when the anglc of incidence of the illuminating beam is small (Stiles and Crawford, 1933). As the angle of incidence increases, a greater proportion of the incident light leaks out of the photoreceptor. Some of the light that leaks out of the photoreceptor inner segments without traversing the photopigment will eventually be caught by the $\mathrm{CCD}$, rcducing the signal-to-noise ratio of the spectral measurements and making the distinction of small spectral sensitivity differences among the $\mathrm{S}, \mathrm{M}$, and $\mathrm{L}$ cones more difficult. To minimize this contrast reduction, bright spots corresponding to light emerging from cone outer segments were brought into focus using IR illumination to prevent photopigment bleaching. The cone optical axes were then aligned with the optical axis of the microscope by tilting the stage until the amount of light being transmitted through the photoreceptors was maximized.

Figure 2 shows that when the tips of the outer segments are in sharp focus and the axes of the photoreceptors are aligned, individual photoreceptors glow brightly. In fact, the intensity of the light emerging from the outer-segment tip was always greater than the intensity of the illuminating beam, showing that both rods and cones have optical gains greater than 1 and as high as 3 . Thus, it is possible in this preparation to funnel incident light efficiently through the photoreceptors in relatively large patches of peripheral retina.

Figure 3 , however, shows that achieving efficient transmission depends critically on the angle of incidence of the illuminating beam relative to the axes of the photoreceptors. As the angle of incidence is increased, transmission through a photoreceptor falls off rapidly, declining by a factor of 3 for angles as small as $3.8^{\circ}$. This decline, measured in fresh retina, corresponds to a Stiles-Crawford angular tuning function with a $\rho$ value of $\sim 0.2$ (full width at half height of $2.4^{\circ}$ ) and is similar to previous measurements made in vitro (for review, see Enoch and Lakshminarayanan, 1991). The details and implications of these measurements will be published separately.

Calculations of axial transmittance. Figure 4 shows how we measured the proportion of light incident on the aperture of the photoreceptor that was not absorbed by photopigment (axial transmittance). Two images of a patch of well aligned retina $\sim 30^{\circ}$ in the periphery were taken with 510 $\mathrm{nm}$ light. The first image (left) was taken with the photopigment largely unbleached, and the photons that were collected are those that were not absorbed as they passed through the photopigment. The second image 


\section{Cone Focus}
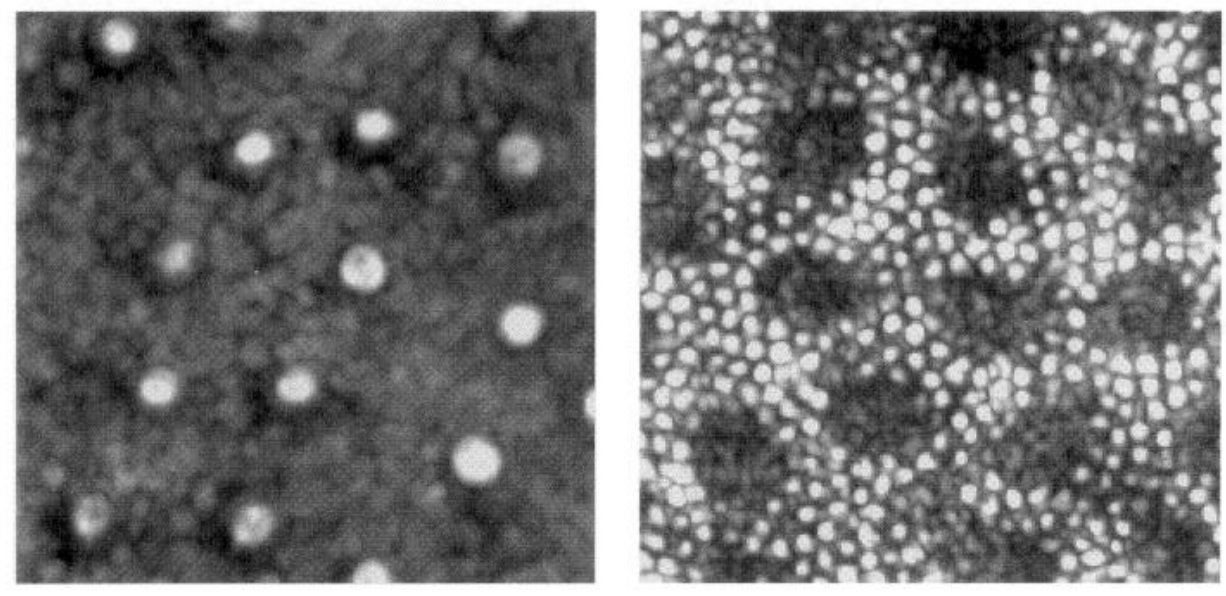

\section{Rod Focus}

Figure 2. Images of photoreceptors that are efficiently funneling $590 \mathrm{~nm}$ light. The bright spots are images of well aligned photoreceptors in two patches of peripheral monkey retina. The cone outer-segment tips (left) end deeper in the retina than the rod outer-segment tips (right) and are rarely in focus simultaneously. The distance between the ends of the rods and cones is $7 \mu \mathrm{m}$ in this patch of retina. (center) was taken after bleaching all of the photopigment, and it represents the total number of photons available to be absorbed. Complete bleaching was accomplished by removing all filters and apertures from the illuminating beam and exposing the retina to the full intensity of the filament for $1 \mathrm{sec}$. The adequacy of the bleach was checked by bleaching a second time and collecting another set of images. Although a change in transmittance was observed before and after the first bleach, no further change was observed after a second $4 \mathrm{sec}$ bleach. The before-bleach image was then divided by the after-bleach image on a pixel by pixel basis. This division factors out all of the contrast attributable to agents such as photostable pigments, which are common to both images, and produces a transmittance image (right), i.e., an image of the proportion of incident light that was not absorbed by the photopigment. In a transmittance image, dark regions represent higher pigment absorptance (1transmittance). Each cone outer segment is a small dark disk (arrow) surrounded by a light ring. This ring is the photopigment-free region attributable to the large diameter difference between the inner and outer segments of peripheral cones. Filling in around the cones are out-of-focus rod outer segments (arrow), which also absorb strongly at $510 \mathrm{~nm}$ and therefore appear quite dark. The lower edge of this image is somewhat unusual in that both rods and cones are in focus simultaneously. Usually, the cones are shorter than the rods.

To calculate the absorptance of each photoreceptor, the absorptances of each pixel making up the image of the outer-segment tip were averaged. This was done separately for images taken at each of the three wavelengths. The photoreceptor was then classified by the type of photopigment it contained (see below). If the photoreceptor was classified as an $\mathrm{M}$ cone, its $510 \mathrm{~nm}$ absorptance was used to calculate the absorptance at the wavelength of maximum absorptance $\left(\lambda_{\max }\right)(530 \mathrm{~nm})$. If the photoreceptor was classified as an L cone, its $590 \mathrm{~nm}$ absorptance was used to calculate its absorptance at the $\lambda_{\max }$ of the $\mathrm{L}$ cone photopigment $(561 \mathrm{~nm})$. If the photoreceptor was a rod, its absorptance at $510 \mathrm{~nm}$ was used to calculate the absorptance at the $\lambda_{\max }$ of rhodopsin $(491 \mathrm{~nm})$. These calculations were made using measurements of macaque photoreceptor spectral sensitivity (Baylor et al., 1987). Only those patches of retina that exhibited high, cone outer-segment densities surrounded by a ring of lower absorptance embedded in a background of high rod absorptance, as illustrated in Figure 4, were analyzed. Within each patch, only those photoreceptors with axial absorptances exceeding 0.2 were included in our sample.

Classification of photoreceptors. To determine the spectral class of each photoreceptor, we compared the transmittance of its photopigment at each of the three wavelengths. For example, at the long wavelength (590 $\mathrm{nm}$ ) that provides maximum contrast between $\mathrm{M}$ and $\mathrm{L}$ cones, $\mathrm{L}$ cones transmit a relatively small fraction of the incident light and appear dark, whereas the $\mathrm{M}$ cones transmit relatively more light and appear brighter.
$-3.8^{\circ}$
$-1.9^{\circ}$
0
$1.9^{\circ}$
3.8
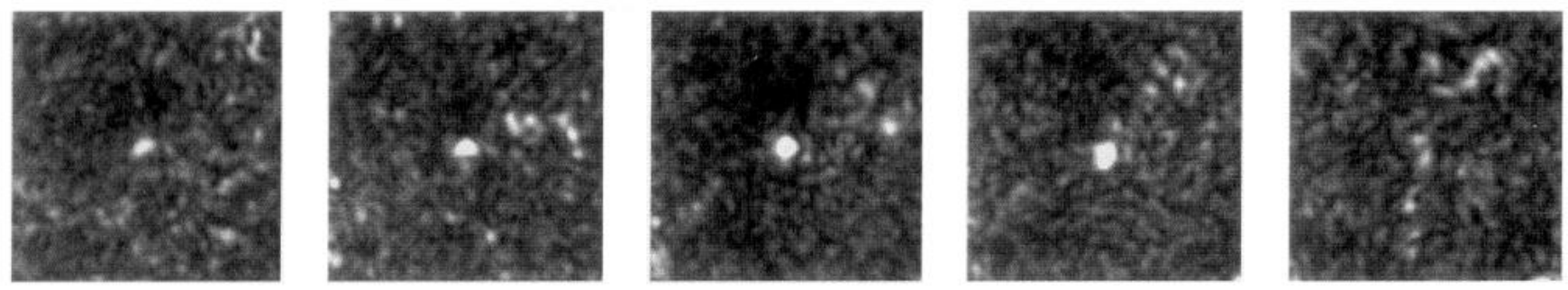

\section{$2 \mu \mathrm{m}$}

Figure 3. Changes in the efficiency of photoreceptor funneling as a function of the angle of incidence of the $600 \mathrm{~nm}$ illuminating beam. A collimated beam was brought to focus on the retina with a lens. A small aperture conjugate with the retina in a collimated portion of the beam was translated to change the angle of incidence. The center image $\left(0^{\circ}\right)$ shows a well aligned cone that is collecting light efficiently. Increasing the angle of incidence to $\pm 1.9^{\circ}$ noticeably reduced the transmission efficiency. By $\pm 3.8^{\circ}$, transmission was reduced by a factor of 3 . 
Figure 4. Calculating photopigment transmittance. Transmittance (right) is calculated by dividing an image of photoreceptors taken before bleaching the photopigment (left) by an image of the same patch of retina taken after bleaching (center). These images were taken with $510 \mathrm{~nm}$ light. Arrows point to a rod outer-segment tip and a cone outersegment tip showing high absorptances. We calculated that at least $2 \times 10^{5}$ photons need to be collected from each outer segment to detect reliably a contrast of $0.5 \%$. This should allow us to discriminate the photopigments. After taking into account the quantum efficiency of the CCD camera, the geometry of the photoreceptor, and the absorption properties of pigment molecules, 6 or 7 $\log$ photons were delivered to each receptor to produce the required photon catch. We calculate that the three imaging exposures bleached $<10 \%$ of the pigment.

\section{Before Bleach}

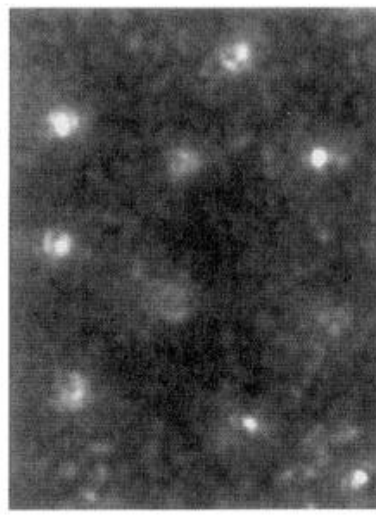

$\overline{5 \mu \mathrm{m}}$
After Bleach

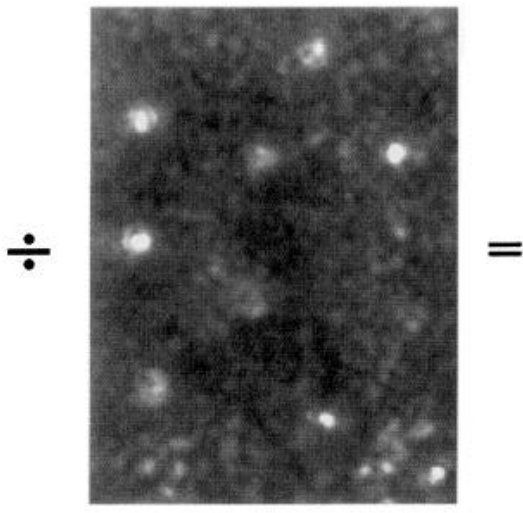

\section{Photopigment} Transmittance

$\mathrm{S}$ cones transmit almost all of the light and appear brightest. By comparing the pattern of transmittances at different wavelengths, the type of photopigment contained in each photoreceptor can be determined.

We used two different formats to illustrate the classification data. The first calculation was applied directly to the three transmittance images. A composite image was produced by determining the weights of the three primaries $(440,510$, and $590 \mathrm{~nm})$ that would sum to white and then scaling each of the three transmittance images so that a transmittance of one at all three wavelengths (no photopigment absorption) would map to white on the Commission Internationale de l'Éclairage (CIE) chromaticity diagram. The chromaticity coordinates of each pixel were then calculated and used to produce a color image. This image shows what the distribution of photopigment would look like if it could be viewed under the microscope with white light without bleaching. The second format was a plot of each photoreceptor in a three-dimensional space with axes that were its photopigment absorptances at each of the three wavelengths. In this space, the rods and cones should lie along lines representing the expected absorptances of the four types of photopigments, as calculated from their spectral sensitivities (Baylor et al., 1987; Schnapf et al., 1988). The rod and cone predictions are lines and not points because this space also represents the amount of photopigment present. A short outer segment with low axial absorptance would map nearer the origin than a long outer segment with higher axial absorptance. Once plotted, a singlelinkage cluster analysis was used to group the data points into two clusters. Each cone was classified by noting whether it was a member of the cluster nearest the $\mathrm{M}$ or $\mathrm{L}$ prediction line.

\section{RESULTS}

\section{Axial photopigment absorptance}

Photopigment transmittance imaging can yield axial absorptances that are many times higher than those measured using axial MSP and that are consistent with measurements made using other techniques. Examples of such rods and cones are labeled with arrows in the transmittance image of Figure 4. Their absorptances are summarized in Table 1.

Mean absorptances for $\mathrm{M}$ and $\mathrm{L}$ cones were 0.28 and 0.30 , respectively. We estimate that cone outer segments at the eccentricity $\left(\sim 30^{\circ}\right)$ of these patches of retina are roughly $10-15 \mu \mathrm{m}$ long, with rods being a few microns longer. The mean absorptances of monkey and human rods were 0.51 and 0.41 . The high absorptances of human rods show that even several hours after death most of the photopigment remains unbleached if it can be shielded from light exposure.

Unfortunately, despite some successes, most patches of retina yielded absorptances that did not exceed 0.05 , as was the case for axial MSP. We found many locations, as shown in Figure 5, in which a bright cone in a raw image of the retina did not correspond to a high absorptance in the transmittance image. Likewise, areas of high absorptance did not always correspond to bright cones in the raw image. Apparently, the outer segments of these cones were bent, as shown in Figure 6, allowing most of the light captured by the inner segment to leak out before traversing the photopigment.

\section{Distinguishing $\mathbf{M}$ from $\mathbf{L}$ cones}

Those photoreceptors that have high axial densities can also be classified on the basis of the photopigment they contain. Figure $7 A$ is an image made from a small patch of monkey retina with very well aligned photoreceptors $30^{\circ}$ into the periphery. The focus is in the plane of the cone outer-segment tips. The transmittance images at each of the three wavelengths were combined into a single color image that shows what the distribution of photopigment would look like if it could be viewed under the microscope with white light without bleaching. An example of each type of photoreceptor is labeled with an arrow. There are five cones and

Table 1. Summary of photoreceptor absorptances at $\lambda_{\max }$

$\begin{array}{lllr}n & \begin{array}{l}\text { Mean } \\ \text { absorptance }\end{array} & \text { SD } & \begin{array}{r}\text { Number } \\ \text { of patches }\end{array} \\ 53 \text { (45 Macaca fascicularis, 8 Macaca mulatta) } & 0.28 & 0.04 & 3 \\ 63 \text { (51 Macaca fascicularis, 12 Macaca mulatta) } & 0.30 & 0.03 & 3 \\ 273 & 0.51 & 0.04 & 13 \\ 183 & 0.41 & 0.03 & 1\end{array}$




\section{Before Bleaching}

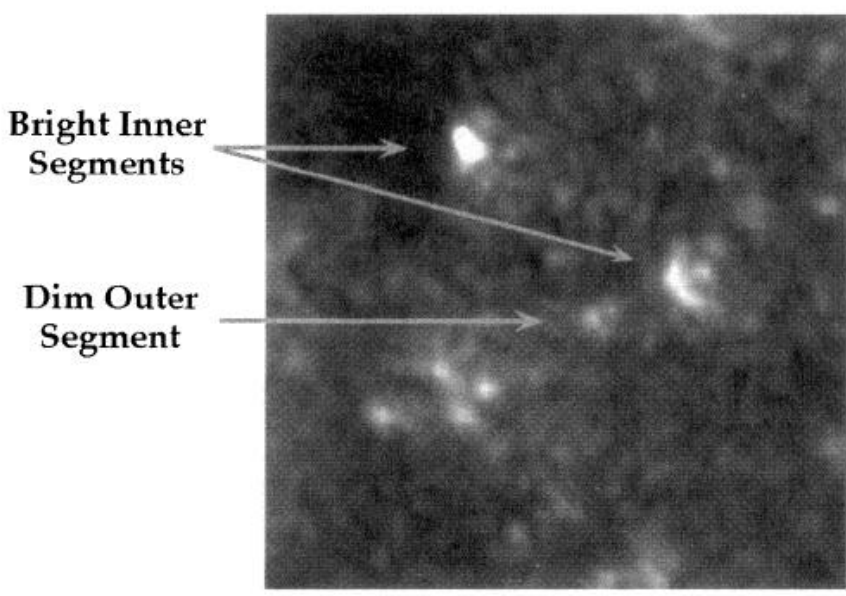

\section{Transmittance}

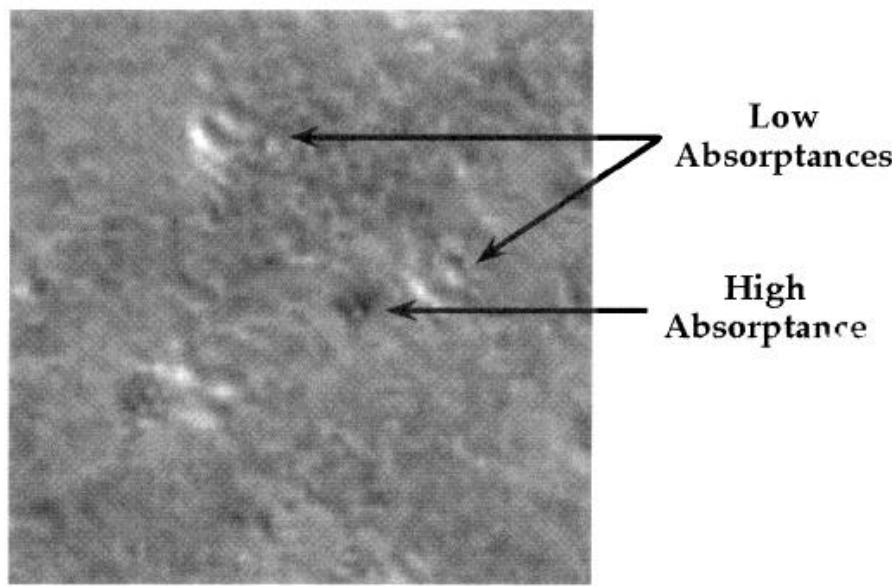

$5 \mu \mathrm{m}$

Figure 5. Images taken with $510 \mathrm{~nm}$ light of cones in a patch of peripheral retina (left) and a transmittance image of the same patch (right). Two cones with bright inner segments (arrows, top left) before bleaching seem to be funneling light efficiently but nevertheless show little photopigment absorptance (arrows, top right). The region of highest absorptance (arrow, middle right) corresponds to a much less prominent spot of light in the unprocessed image (arrow, middle left).

many rods in this patch of retina. The ends of rod outer segments are small wine-colored disks when they are in good focus and a more diffuse wine color when they are not. Each cone outer segment is surrounded by a relatively desaturated ring corresponding to the pigment-free inner segment. Of the five cone outer segments, three are blue and two are purple. The blue disks are L cones, and the purple disks are M cones. As a check, we also calculated the expected hues of the photopigments by looking up their transmittances at the three wavelengths corresponding to the primaries that we used, calculating the CIE chromaticity coordinates of a white light constructed from these primaries and filtered by these photopigments, and then finding the corresponding hues on the spectrum locus. The hues in the composite image are close to the predicted values.

Figure $7 B$ is a photopigment transmittance image of a larger patch of retina from another animal. This image shows that even

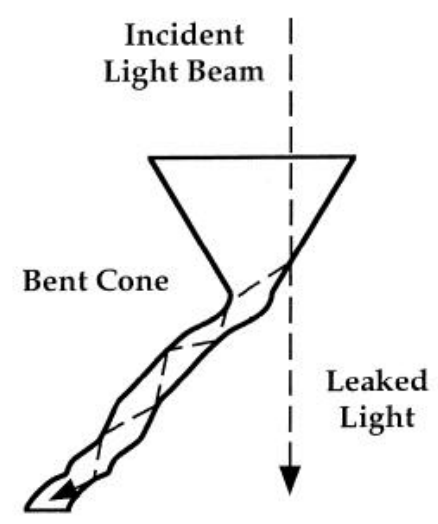

Figure 6. A schematic drawing of incident light leaking out of a bent photoreceptor before traversing the photopigment. The leaked light forms a bright spot exhibiting low absorptance. The small amount of light that does get coupled into the outer segment forms a dimmer offset spot that exhibits high absorptance. in peripheral retina where the numerical density of cones is relatively low, it is possible in the best cases to image simultaneously patches of retina containing $\sim 100$ cones and thousands of rods. This image was optimized for cone outer-segment focus. In the right center of the image, however, the rods are reasonably distinct. The hue of individual cones is hard to discern by eye at this lower magnification, but the spectral signature of each cone can be calculated reliably from the photon catches of the pixels that make up the image of the outer-segment tip (see below). Figure $7 C$ is the same image shown in Figure $7 B$, with the addition of colored markers to show the results of the spectral classification. Only those cones that had absorptances of 0.20 or higher for at least one of the three wavelengths are labeled. Most of the cones that failed to meet this criterion were located along the top and bottom of the image in areas of softer focus. Although several $\mathrm{S}$ cones would be expected in a sample of this size, none of the cones in this sample fell near the predicted absorptances for $\mathrm{S}$ cones. There were a few photoreceptors such as those just to the right of the question marks that had a yellowish hue similar to that expected from $\mathrm{S}$ cones; however, their absorptances were not high enough for reliable classification. Finally, although this image is not extensive enough for a detailed analysis, it is obvious that the packing geometry of the $\mathrm{M}$ and $\mathrm{L}$ cone submosaics is not crystalline.

The photoreceptors labeled in Figure $7 C$ were classified spectrally by plotting their positions in a three-dimensional space with axes that are their absorptances at 440,510 , and $590 \mathrm{~nm}$. All of the monkey photoreceptors summarized in Table 1 are plotted in Figure $8 A$. For simplicity, the $440 \mathrm{~nm}$ dimension has been omitted because $\mathrm{L}$ and $\mathrm{M}$ cones and rods are distinguishable on the basis of 510 and $590 \mathrm{~nm}$ alone. The solid lines (that extend back through the origin) are the calculated positions of $\mathrm{L}$ cones, $\mathrm{M}$ cones, and rods based on published photopigment transmittance spectra (Baylor et al., 1987; Schnapf et al., 1987, 1988). A singlelinkage cluster analysis divided the cone photoreceptors into two 

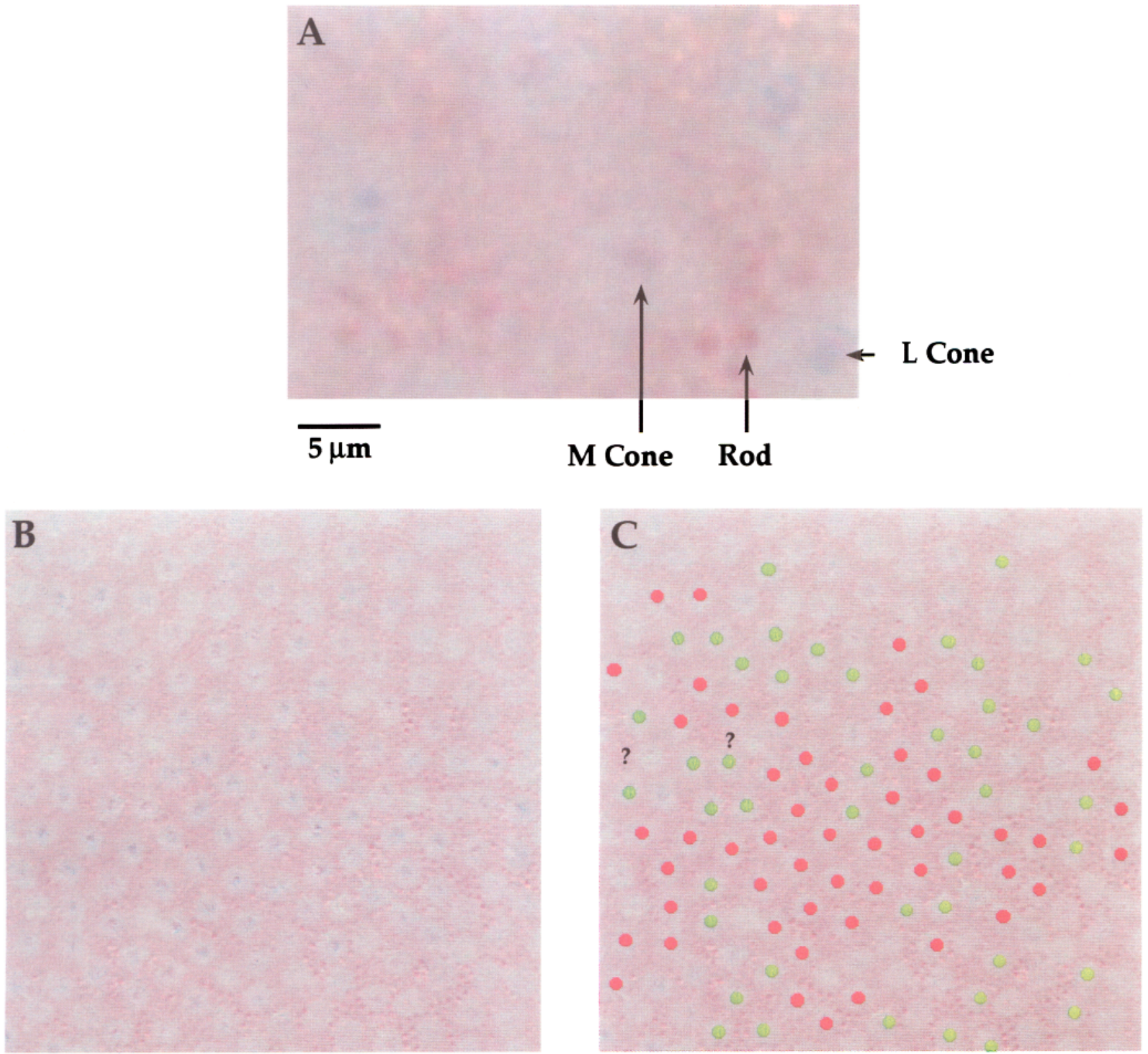

$\overline{20 \mu \mathrm{m}}$

Figure 7. Photopigment transmittance images of peripheral macaque (M. fascicularis) retina as they would look in white light if the photopigment did not bleach. $A$, This patch, shown at high magnification, contains five cones and numerous rods. The blue disks are $\mathrm{L}$ cones, and the purple disks are $\mathrm{M}$ cones. The rods filling in around the cones are wine-colored. B, A larger patch of peripheral retina at lower magnification illustrating the large numbers of photoreceptors that can be imaged simultaneously. At this magnification, the low absorptance ring around each cone is obvious. The top and bottom edges of the image are out of focus. $C$, After graphical classification, the cones of image $B$ are overlaid with colored dots to make their identity obvious $($ red $=\mathrm{L}$ cone, green $=\mathrm{M}$ cone). The question marks are just to the left of two cones with a yellowish hue similar to that expected of $\mathrm{S}$ cones. Cones with peak absorptances of $<0.20$ are not marked.

clusters on the basis of their 510 and $590 \mathrm{~nm}$ absorptances. These clusters were identical to those identified visually by the authors before the analysis was performed. Both the $\mathrm{L}$ and $\mathrm{M}$ cone clusters are elongated in the direction of the prediction lines, as would be expected if they had small differences in axial absorptance attributable to slight variability in outer-segment length or photopigment density. In Figure $8 B$, the visual effect of these differences in axial absorptance has been minimized by plotting a histogram of the log of the $510 / 590 \mathrm{~nm}$ absorptance ratio of each cone. Again, the cones tend to cluster into two groups, but it is obvious that they do not fall exactly on the prediction lines. Calculations predict a $\log 590 / 510$ absorptance ratio of -0.14 for $\mathrm{L}$ cones and 0.39 for $\mathrm{M}$ cones, whereas the measured mean values are approximately -0.07 for $\mathrm{L}$ cones and 0.22 for $\mathrm{M}$ cones. Although we are not yet sure why that is, several possibilities are addressed in the Discussion. Finally, it is worth noting that al- 


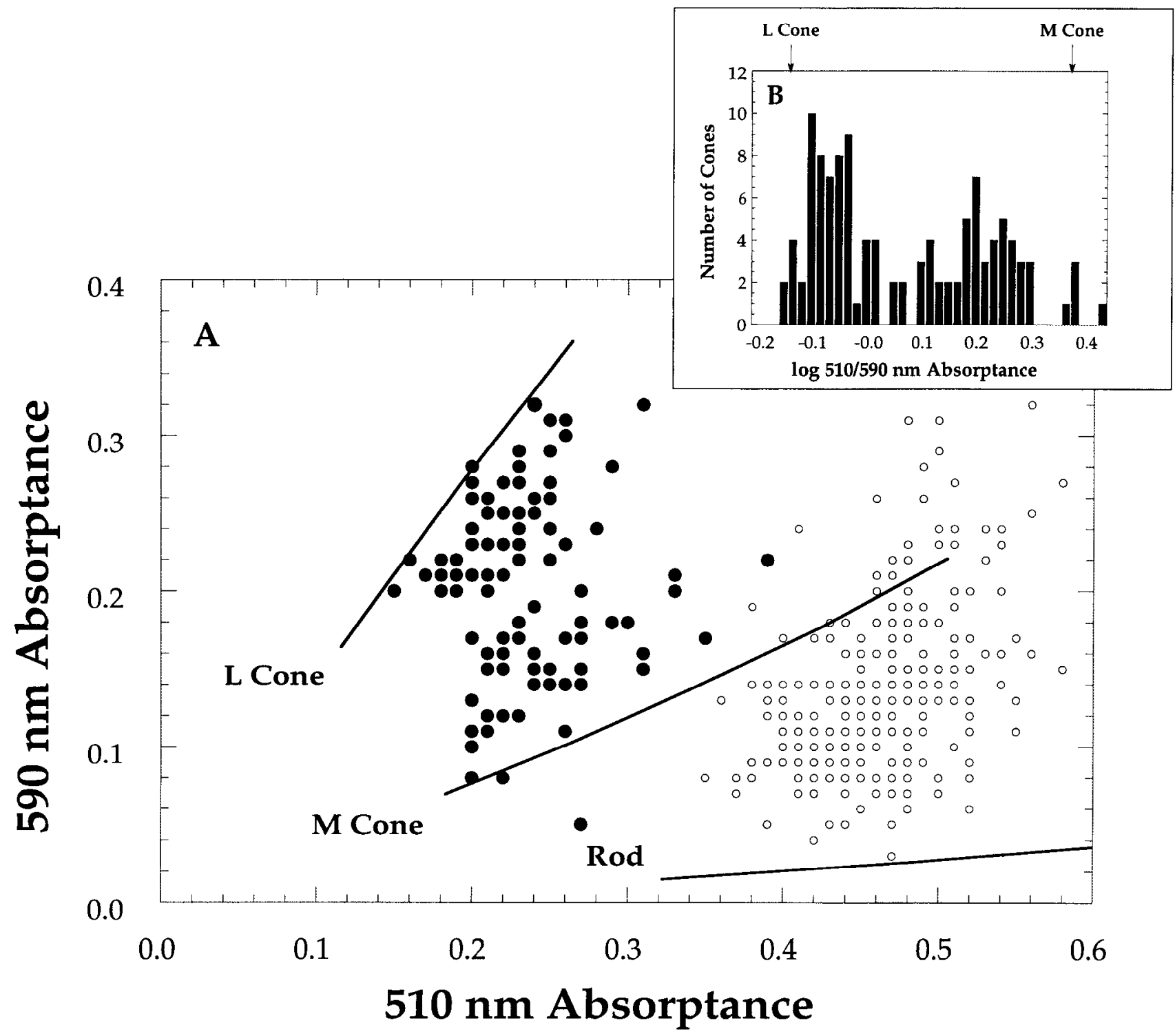

Figure 8. The graphical classification of monkey photoreceptors. A, The absorptances at $590 \mathrm{~nm}$ of each of the monkey photoreceptors summarized in Table 1 are plotted against their absorptances at $510 \mathrm{~nm}$. The solid lines, which actually extend back through the origin, are the expected positions of $\mathrm{L}$ cones, $M$ cones, and rods determined based on spectral sensitivity measurements. The lines were calculated from the cquation $f(\lambda)=1-10\left(a(\lambda)^{*} b\right)$, where $f(\lambda)$ is axial absorptance at wavelength $\lambda, a(\lambda)$ is the relative spectral sensitivity (Baylor et al., 1987, their Eqs. 3 and 6 ), and $b$ is axial photopigment density. Filled circles are cones, and open circles are rods. $B$, A histogram of the log of the $510 / 590 \mathrm{~nm}$ absorptance ratio for the cones summarized in Table 1. The labeled arrows show the expected position of the $\mathrm{L}$ and $\mathrm{M}$ cones as calculated in $A$.

though the rod and $M$ cone clusters are close together, they are also separated by 0.17 on the $440 \mathrm{~nm}$ axis (not shown) and can always be distinguished on morphological criteria.

Once the cones were classified by photopigment type, we also analyzed the relative numbers of the two cone types and their assignment to positions in the cone lattice. Of the 116 cones plotted, $54 \%$ were classified as $\mathrm{L}$ cones, a percentage that is consistent $(p=0.23)$ with a population having equal numbers of $\mathrm{I}$, and $\mathrm{M}$ cones but not with a population having two I, cones for every $\mathrm{M}$ cone $(p=0.002)$.

We also analyzed the assignment of the $M$ and $L$ cones of Figure $7 C$ into submosaics using a nearest-neighbor analysis (Shapiro et al., 1985; Curcio et al., 1991) in which the proportions of $M$ or $L$ cone nearest-neighbors that were themselves $M$ or $L$ cones were tabulated and compared to the distribution produced by randomly assigning $(n=10,000) \mathrm{M}$ and $\mathrm{L}$ cones to the lattice of Figure $7 C$. Cones in the sample had a significantly higher proportion $(p=0.006)$ of neighbors of like-type than would be expected from a random distribution. A sample proportion less than the mean of the random distribution indicates more regular spacing than would be expected in a cone mosaic with $\mathrm{M}$ and $\mathrm{L}$ cones that are randomly assigned, whereas a sample proportion greater than the mean indicates more clumping.

\section{DISCUSSION}

Photopigment transmittance imaging can quickly and simultaneously classify photoreceptors in fresh, excised primate retina directly on the basis of their absorptance spectra. In those patches of peripheral macaque retina in which photoreceptors remain well aligned, appropriately high axial photopigment absorptances can 
be measured, and the cones cluster into two spectral groups corresponding to the $\mathrm{M}$ and $\mathrm{L}$ cones.

Photopigment transmittance imaging has substantial advantages over other techniques for measuring photoreceptor topography. The first advantage is the ability to measure many photoreceptors simultaneously, unlike sequential techniques such as MSP (Bowmaker and Dartnall, 1980; Bowmaker et al., 1980a,b, 1983, 1991; Dartnall et al., 1983a,b; MacNichol et al., 1983; Harosi, 1987), suction-electrode recording (Baylor et al., 1987; Schnapf et al., 1987, 1988), and small-spot stimulation (Williams et al., 1981).

Second, the technique classifies photoreceptors on the basis of their photopigment spectra. Some indirect techniques have provided verification of the link to photopigment type (Stafford et al., 1994), but others have not (Marc and Sperling, 1977; Ahnelt et al., 1987). Similarly, in psychophysical small-spot detection experiments (Walraven, 1974; Smith and Pokorny, 1975; Cicerone and Nerger, 1989; Vimal et al., 1989; Wesner et al., 1991; Cicerone and Otake, 1993; Gowdy et al., 1993; Cicerone et al., 1994), assumptions underlying the detection model are difficult to check.

Finally, the technique preserves retinal topography and viability better than transverse MSP, suction-electrode recording, and current molecular techniques (Hagstrom et al., 1994, 1995). Someday it may be possible to identify photoreceptors using this technique and then study retinal circuitry in the same patch physiologically (Dacey and Lee, 1994a,b) or anatomically (Calkins et al., 1994).

In those patches of retina in which it worked well, photopigment transmittance imaging had the advantages of axial MSP without its low axial absorptance. Recent absorptance measurements using color matching and retinal densitometry (Burns and Elsner, 1985; Burns et al., 1987; van Norren and van de Kraats, 1989; Elsner et al., 1993) range from 0.35 to 0.68 at the fovea and decrease with increasing eccentricity. Our mean cone absorptance of 0.3 for peripheral retina is consistent with these data. Assuming a specific photopigment density of $0.016 \mathrm{log}$ units $/ \mu \mathrm{m}$, a cone outer segment estimated to be $10-15 \mu \mathrm{m}$ long should have an absorptance at $\lambda_{\max }$ between 0.31 and 0.42 . Rods would be slightly higher. Our measurements fall at the lower end of this range, suggesting that the outer segments are intact and stray light is insignificant.

Even when the technique works less well, the data suggest that the low photopigment absorptances reported by axial MSP may be attributable to (1) artifactual bending of receptors and (2) difficulty in locating outer-segment tips. When photoreceptors bend (Fig. 6), only an imaging detector allows an extended region to be searched for the high absorptances corresponding to outersegment tips. Imaging and careful alignment clearly increase the number of receptors for which high photopigment absorptances can be measured and for which reliable spectral classifications can be made. Nevertheless, because small misalignments of the photoreceptors can reduce substantially the signal-to-noise ratio of the measurements, a major limitation on the yield is the ability to keep receptors straight and well aligned. This is especially true for foveal cones. Therefore, caution must be used when interpreting the optical properties of cones viewed in anatomical preparations. Even bent cones that leak light can be imaged as bright spots. Their waveguide modal patterns, however, may not reflect accurately the optical properties of the retina in vivo.

Although our data are consistent with equal numbers of $\mathrm{L}$ and $M$ cones in the macaque retina, the true L-to-M ratio is not known accurately. Ratios reported by MSP and suction-electrode studies
(Brown and Wald, 1963, 1964; MacNichol, 1964; Marks et al., 1964; Bowmaker and Dartnall, 1980; Bowmaker et al., 1980a,b, 1983, 1991; Dartnall et al., 1983a,b; MacNichol et al., 1983; Baylor et al., 1987; Harosi, 1987; Schnapf et al., 1987, 1988; Mollon and Bowmaker, 1992) range from 0.5 to 6 , likely attributable to the small numbers of photoreceptors measured in single locations of individual retinas. Pooling data across locations, across different studies, and even across similar species can increase the sample size, but only at the expense of information about individual variability, species differences, and retinal homogeneity.

The assignment of $\mathrm{L}$ and $\mathrm{M}$ photopigments to cones (Fig. 7C) is not highly regular. Rather, cones tended to clump with other cones of the same type. A single axial MSP study (Mollon and Bowmaker, 1992) based on a few very small clusters of cone outer segments concluded that photopigment assignment could not be distinguished from random assignment.

In short, photopigment transmittance imaging can answer questions about retinal topography. Nevertheless, although the patch of retina imaged in Figure $7 C$ represents the largest single sample of photoreceptors ever classified, more detailed data will be necessary to determine the true range of L-to-M cone ratios and confirm that $\mathrm{L}$ and $\mathrm{M}$ cones clump.

Three other issues require further explanation. First, we have not identified any $S$ cones. Dacey and Lee (1994a,b) showed that retinal ganglion cells, maintained in vitro, receive $\mathrm{S}$ cone input, so there is apparently no incompatibility between tissue culture and $\mathrm{S}$ cone viability, although removing the pigment epithelium could cause damage. If $\mathrm{S}$ cone outer segments are longer or shorter than average, our attempts to bring the largest numbers of cones into best focus may have excluded them. Finally, short-wavelengthabsorbing photoproducts might reduce $S$ cone absorptance,

Second, measured spectral absorptances do not match predictions made on the basis of suction-electrode recordings (Baylor et al., 1987; Schnapf et al., 1987, 1988). The L cones (Fig. 8) show decreased absorptance at $590 \mathrm{~nm}$ relative to their absorptance at $510 \mathrm{~nm}$, whereas $M$ cones and rods show the reverse. To match the data and the predictions, the $\lambda_{\max }$ of $\mathrm{L}$ and $\mathrm{M}$ cones would need to be shifted from 561 to $553 \mathrm{~nm}$ and from 531 to $540 \mathrm{nIm}$, respectively. These are larger shifts than the $4.3 \mathrm{~nm}$ range of $\lambda_{\max }$ of cones of a single class reported by Schnapf et al. (1988). Because photopigment transmittance is the ratio of two images, our photopigment spectra are not subject to distortion by photostable filters. Other explanations can be eliminated because they can only shift the sensitivities of $M$ and $L$ cones in the same direction. For example, fluorescence induced by the illuminating beam of any retinal structure except the photopigment itself would reduce measured photopigment absorptance. If the intensity of fluorescence varied with wavelength, the ratios of 510 to $590 \mathrm{~nm}$ absorptance might change, but in exactly the same way for both $\mathrm{M}$ and $\mathrm{L}$ cones. Spectral distortions caused by partial bleaching can be ruled out on similar grounds.

Although Liebman (1972) concludes that interference from blcaching photoproducts is not a problem for axial MSP, our technique is sensitive to interference because it uses an image of fully bleached retina as a reference. Photoproduct interference could shift the spectra toward one another, however, only if stable photoproducts absorb at wavelengths both above the $\lambda_{\max }$ of the $\mathrm{L}$ cones and below the $\lambda_{\max }$ of the $\mathrm{M}$ cones. Because most photoproducts absorb strongly at short wavelengths, both cone spectra should be shifted toward longer wavelengths. The shift of rods toward longer wavelengths is possible, although high axial absorptances suggest that the photoproducts have little ab- 
sorptance at or above $510 \mathrm{~nm}$. High axial absorptances suggest that light scatter is neither severe nor a mechanism for spectral contamination. The wide spacing and sparseness of peripheral cones minimize cross-contamination of both neighboring cones and rods. Contamination of cone spectra by nearby rods would not move the $\mathrm{M}$ and $\mathrm{L}$ cone spectra toward each other. There is no evidence from genetic studies (Hagstrom et al., 1994) that photopigments are mixed within individual photoreceptors and mixing could not explain the shift in rod spectra. Perhaps the spectral differences are attributable to wavelength dependencies of the waveguides or shifts in photopigment spectral sensitivity in vitro.

Last, the large spread of the $L$ and $M$ cone clusters of Figure 8 suggests remaining sources of measurement noise over and above sinall differences in the true $\lambda_{\max }$ of the photopigments. Individual $M$ cones have values of $\lambda_{\max }$ ranging between 519 and $547 \mathrm{~nm}$, whereas individual $\mathrm{L}$ cones have values ranging between 548 and $562 \mathrm{~nm}$. Variations in outer-segment length and pigment concentration shift the data parallel to the prediction lines and cannot contribute to these large ranges. Additionally, the cooled CCD camera has low intrinsic noise, and sufficient photons are collected to detect absorptance differences as small as $0.5 \%$. It seems likely that the primary remaining source of noise is retinal, in the form of changes in photoreceptor alignment as a result of mechanical or osmotic pressure, or changes in the optical properties of the photoreceptors that affect the confinement of light. The ultimate stability of the technique depends on damping local changes.

\section{REFERENCES}

Ahnelt PK, Kolb H, Pfiug R (1987) Identification of a subtype of cone photoreceptor, likely to be blue sensitive, in the human retina. $J$ Comp Neurol 255:18-34.

Alperi M, Madseidvady F, Ohba N (1971) The kinetics of cone visual pigments in man. Vision Res 11:539--549.

Ames A, Nesbett FB (1981) In vitro retina as an experimental model of the central nervous system. J Neurochem 37:867-877.

Baylor DA, Nunn BJ, Schnapf JL (1987) Spectral sensitivity of cones of the monkey Macaca fascicularis. J Physiol (Lond) 390:145-160.

Bowmaker JK, Dartnall HJA (1980) Visual pigments of rods and cones in human retina. J Physiol (Lond) 298:501-511.

Bowmaker IK, Dartnall H.JA, Mollon ID (1980a) Microspectrophotometric demonstration of four classes of photoreceptor in an old world primate, Macaca fascicularis. J Physiol (Lond) 298:131-143.

Bowmaker JK, Dartnall HJ , Lythgoe JN, Mollon JD (1980b) The visual pigments of rods and cones in the rhesus monkey, Macaca mulatta. J Physiol (Lond) 274:329-348.

Bowmaker JK, Mollon JD, Jacobs GH (1983) Microspectrophotometric results for old and new world primates. In: Colour vision (Mollon JD, Sharpe LT, eds), pp 57-68. London: Academic.

Bowmaker JK, Astell S, Hunt DM, Mollon JD (1991) Photosensitive and photostable pigments in the retinae of old world monkeys. $J$ Exp Biol 156:1-19.

Brown PK, Wald G (1963) Visual pigments in human and monkey retinas. Nature 200:37-43.

Brown PK, Wald G (1964) Visual pigments in single rods and cones of the human retina. Science $144: 45-52$.

Burns SA, Elsner AE (1985) Color matching at high illuminances: the color match area effect and photopigment bleaching. J Opt Soc Am [A] 2:698-704.

Burns SA, Elsner AE, Lobes LA, Doft BH (1987) A psychophysical technique for measuring cone photopigment bleaching. Invest Ophthalmol Vis Sci 28:711-717.

Calkins DJ, Schein SJ, Tsukamoto Y, Sterling P (1994) M and L cones in macaque fovea connect to midget ganglion cells by different numbers of excitatory synapses. Nature 371:70-72.

Cicerone CM, Nerger JL (1989) The relative numbers of longwavelength-sensitive to middle-wavelength-sensitive cones in the human fovea centralis. Vision Res 29:115-128.
Cicerone CM, Otake S (1993) Placement of $\mathrm{L}$ and $\mathrm{M}$ cones in the peripheral photorcceptor matrix. Invest Ophthalmol Vis Sci [Suppl] 34:1042.

Cicerone CM, Gowdy PD, Otake S (1994) Composition and arrangement of the cone mosaic in the living human eye. Invest Ophthalmol Vis Sci [Suppl] 35:1471.

Curcio CA, Allen K, Sloan K, Lerea C, Hurley J, Klock I, Milam A (1991) Distribution and morphology of human cone photoreceptors stained with anti-bluc opsin. J Comp Neurol 312:610-624.

Dacey DM (1989) Axon-bearing amacrine cells of the macaque monkey retina. J Comp Neurol 284:275-293.

Dacey DM, I ee BB (1994a) Physiology of identified ganglion cell types in an in vitro preparation of macaque retina. Invest Ophthalmol Vis Sci [Suppl] 35:3449.

Daccy DM, Lcc BB (1994b) The 'bluc-on' opponcnt pathway in primatc retina originates from a distinct bistratified ganglion cell type. Nature 367:731-735.

Dartnall HJA, Bowmaker JK, Mollon JD (1983a) Human visual pigments: microspectrophotometric results from the eyes of seven persons. Proc R Soc Lond [Biol] 220:115-133.

Dartnall HJA, Bowmaker JK, Mollon JD (1983b) Microspectrophotometry of human photoreceptors. In: Colour vision (Mollon JD, Sharpe LT, eds), pp 69-80. London: Academic.

de Monasterio FM, McCrane EP, Newlander JK, Schein SJ (1985) Density profile of blue-sensitive cones along the horizontal meridian of macaque retina. Invest Ophthalmol Vis Sci 26:289-302.

Denton EJ, Wyllic JH (1955) Study of the photosensitive pigments in the pink and green rods of the frog. J Physiol (Lond) 127:81-89.

Elsner AE, Burns SA, Wobb RH (1993) Mapping cone photopigment optical density. J Opt Soc Am [A] 10:52-58.

Enoch JM, Glismann LE (1966) Physical and optical changes in excised retinal tissue. Resolution of retinal receptors as a fiber optic bundle. Invest Ophthalmol 5:208-221.

Enoch JM, Lakshminarayanan V (1991) Retinal fiber optics. In: Vision and visual dysfunction, Vol I (Cronly-Dillon J, ed), pp 280-309. Boca Raton, FL: CRC.

Gowdy PD, Cicerone CM, Olake S (1993) Foveal L and M cone ratio and topography estimated with chromatic hyperacuity stimuli. Invest Ophthalmol Vis Sci [Suppl] 34:235.

Hagstrom SA, Neitz J, Neitz M, Trusk TC (1994) Characterizing the photopigment genes expressed in individual human cone photoreceptors. Invest Ophthalmol Vis Sci [Suppl] 35:2123.

Harosi FI (1987) Cynomolgus and rhesus monkey visual pigments. J Gen Physiol 89:717-743.

Liebman PA (1972) Microspectrophotometry of photoreceptors. In: Handbook of sensory physiology, Vol VII/1, Photochemistry of vision (Dartnall HJA, ed), pp 481-528. New York: Springer.

MacNichol EF (1964) Retinal mechanisms of color vision. Vision Res 4:119-133.

MacNichol EF, Levine JS, Mansfield RJW, Lipetz LE, Collins BA (1983) Microspectrophotometry of visual pigments in primate photoreceptors. In: Colour vision (Mollon JD, Sharpe LT, eds), pp 13-38. London: Academic.

Marc RE, Sperling HG (1977) Chromatic organization of primate cones. Science 196:454-456.

Marks WB, Dobclle WH, MacNichol EF (1964) Visual pigments of single primate cones. Science 143:1181-1183.

Mollon JD, Bowmaker JK (1992) The spatial arrangement of cones in the primate fovea. Nature 360:677-679.

Ohzu H, Enoch JM (1972) Optical modulation by the isolated human fovea. Vision Res 12:245-251.

Ohzu H, Enoch JM, O'Hair JC (1972) Optical modulation by the isolated retina and retinal receptors. Vision Res 12:231-244.

Ripps H, Weale RA (1963) Cone pigments in the normal human fovea. Vision Res 3:531-543.

Rushton WAH, Henry GH (1968) Bleaching and regeneration of cone pigments in man. Vision Res 8:617-631.

Schnapf JL, Kraft TW, Baylor DA (1987) Spectral sensitivity of human cone photoreceptors. Nature 325:439-441.

Schnapf JL, Kraft 'IW, Nunn BJ, Baylor DA (1988) Spectral sensitivity of primate photoreceptors. Vis Neurosci 1:255-261.

Shapiro MB, Schein SJ, de Monasterio FM (1985) Regularity and structure of the spatial pattern of blue cones of macaque retina. J Am Stat Assoc 80:803-812. 
Smith VC, Pokorny J (1975) Spectral sensitivity of the foveal cone photoreceptors between 400 and $500 \mathrm{~nm}$. Vision Res 12:2059-2071.

Smith VC, Pokorny J, van Norren D (1983) Densitometric measurements of human cone photopigment kinetics. Vision Res 23:517-524.

Stafford D, Marshak D, Jacoby R, Kouyama N (1994) Blue cone bipolar cells of the macaque retina. Invest Ophthalmol Vis Sci [Suppl] 35:1909.

Stiles WS, Crawford BH (1933) The luminous efficiency of rays entering the eye pupil at different points. Proc R Soc Lond [Biol] 112:428.

van Norren D, van de Kraats J (1989) Retinal densitometer with the size of a fundus camera. Vision Res 29:369-374.

Vimal RLO, Pokorny J, Smith VC, Shevell SK (1989) Foveal cone thresholds. Vision Res 29:61-78.
Walraven PL (1974) A closer look at the tritanopic convergence point. Vision Res 14:1339-1343.

Wesner MF, Pokorny J, Shevell SK, Smith VC (1991) Foveal conc detection statistics in color-normals and dichromats. Vision Res 31:1021-1037.

Wikler KC, Rakic P (1990) Distribution of photoreceptor subtypes in the retina of diurnal and nocturnal primates. J Neurosci 10:3390-3401.

Williams DR, MacLeod DIA, Hayhoe MM (1981) Punctate sensitivity of the blue-sensitive mechanism. Vision Res 21:1357-1375.

Yuodelis C, Hendrickson A (1986) A qualitative and quantitative analysis of the human fovea during development. Vision Res 26:847856. 